\title{
Le syndrome parkinsonien du patient âgé dans tous ses états !
}

\section{Stages of parkinsonism in the elderly!}

\author{
M. Verny \\ C) Springer-Verlag France 2012
}

Les difficultés existantes autour du diagnostic des syndromes parkinsoniens dans la population âgée sont nombreuses et leur prise en charge est complexe. Il s'agit d'une problématique fréquente et comme souvent dans cette tranche d'âge, les difficultés sont plus grandes et cela est fréquemment lié aux pathologies associées, dont les lésions vasculaires. L'article de S. Greffard et al. insiste sur le raisonnement à mettre en place pour approcher le diagnostic étiologique d'un syndrome parkinsonien associé à un trouble cognitif, situation fréquente chez les patients âgés. Cet article permet d'insister sur l'importance des caractéristiques cliniques et la nécessité de réaliser une neuro-imagerie dans cette population. L'importance des étiologies de syndrome parkinsonien d'étiologie non-neurodégénérative est soulignée. L'épidémiologie montre que l'incidence et la prévalence de la maladie de Parkinson augmentent avec l'âge. G. Fénelon et J.-P. Brandel, nous proposent d'examiner les différences potentielles entre la maladie de Parkinson des sujets jeunes et des plus âgés. Le diagnostic clinique peut être plus difficile du fait de la fréquence et précocité des signes axiaux dans la population âgée. Parmi les manifestations les plus redoutables, il y a les troubles de l'équilibre avec des chutes et les difficultés cognitives et psychiques. En revanche, les fluctuations motrices et les mouvements anormaux involontaires sont plus rares. Là aussi, la neuro-imagerie est importante pour mettre en évidence, notamment, des lésions vasculaires qui peuvent considérablement interférer avec l'évolution et la sensibilité à la L-dopa. La fréquence et l'importance de la pathologie iatrogène sont majeures chez les patients âgés. Cet aspect est traité dans l'article de A.-M. Bonnet et al. Cela permet aussi de souligner la richesse de la sémiologie des mouvements anormaux liée aux traitements et celle des molécules en cause. Quant aux ressources thérapeutiques au cours de la maladie de Parkin-

\footnotetext{
M. Verny $(\square)$

UPMC (Paris 6), Centre de gériatrie, pavillon M. Bottard,

hôpital Pitié-Salpêtrière, 47-83, boulevard de l'hôpital,

F-75013 Paris, France

e-mail : marc.verny@psl.aphp.fr
}

son, elles sont envisagées dans l'article de E. Roze qui insiste sur le recours quasi exclusif à la L-dopa, l'entacapone et la rasagiline. À des stades avancés de la maladie, les traitements tel que l'apomorphine sous cutanée ou la L-dopa intraduodénale sont à évoquer, même si dans le grand âge, et alors que les comorbidités et les troubles cognitifs sont fréquents, leur utilisation sera difficile. La paralysie supranucléaire progressive (PSP) est d'autant plus importante à connaître que son incidence dans la population âgée à très âgée est relativement importante et n'est donc pas rencontrée de manière exceptionnelle. R. Levy insiste sur la multiplicité des présentations cliniques avec des frontières difficiles avec la dégénérescence corticobasale. Les différents aspects des atrophies multisystématisées (AMS) sont décrits par P. Damier. Toutefois, le diagnostic est particulièrement délicat voire impossible chez les patients âgés comorbides. Cela est encore plus vrai quand il s'agit de cas où les troubles moteurs s'associent à une atteinte cognitive qui, si elle est rare, est bien identifiée maintenant. Enfin, V. Cochen nous propose de nous intéresser aux troubles du sommeil fréquemment associés au syndrome parkinsonien. Cela est d'autant plus important que des ressources thérapeutiques existent et peuvent améliorer de manière significative les patients.

$\mathrm{Au}$ total, on peut retenir que seule une bonne connaissance sémiologique des signes parkinsoniens permet de discuter de manière efficace des différentes hypothèses diagnostiques étiologiques, même si cela est habituellement plus complexe dans la population âgée. Il ne faut pas sous estimer la fréquence des pathologies non neurodégénératives et le recours systématique à des examens de neuro-imagerie est indispensable dans cette population aussi bien pour le diagnostic positif que différentiel ou pour aider à fixer le pronostic. La recherche de la iatrogénie doit être une préoccupation permanente et tous les dictionnaires ou thésaurus de médicaments sont des « grands amis » du gériatre. Quant aux thérapeutiques utilisables dans le grand âge et aux stades avancées de la maladie de Parkinson, il faut les connaître et surtout toujours réfléchir au cas par cas et dans une optique de rapport bénéfice-risque. Est-il utile et raisonnable 
d'améliorer significativement la motricité d'un patient parkinsonien dément avec, pour prix à payer, une majoration de la confusion et un risque fort de chutes traumatisantes ? Le diagnostic des affections comme la PSP et l'AMS doit être fait chaque fois que cela est possible pour fixer le plan de soins, éviter des traitements inutiles et nocifs et fixer autant que possible le pronostic. Enfin, une bonne connaissance des perturbations fréquentes du sommeil permettra la mise en œuvre de traitement améliorant significativement la qualité de vie des patients et de leur entourage. 